\title{
The Extragalactic Infrared Background and its Cosmological Implications - Impressions and Reflections
}

\author{
Malcolm S. Longair \\ Cavendish Astrophysics Group, \\ Cavendish Laboratory, \\ Madingley Road, \\ Cambridge CB3 $O H E$.
}

\begin{abstract}
Many of the key issues facing astrophysicists and cosmologists can be uniquely addressed by observations in near and far infrared, millimetre and submillimetre wavebands. Some highlights of IAU Symposium No. 204 are reviewed and likely future directions of observation, interpretation and theory are discussed.
\end{abstract}

\section{Introduction}

This has been a most enjoyable symposium and one which indicates clearly some of the most important future directions for the study of key cosmological problems. In this brief summary, I will cover the following topics: (i) ancient history and things to remember, (ii) highlights of the new observations of the background radiation in the infrared to millimetre wavebands, (iii) the number counts of objects in these wavebands and the role of modelling, (iv) the Harwit diagram and its extension, (v) an analytic approach to the cosmic star formation rate, and (vi) future directions of observation, interpretation and theory.

\section{Ancient History and Things to Remember}

In the 1960s, we used to joke that observations and interpretations of the background radiation were 'poor man's astronomy', in the sense that the observations could be carried out at low angular resolution with relatively simple telescopes. There was plenty of scope for imaginative theory. This was a time when the rather tricky experiments to determine the long-wavelength radio background emission had given a reasonable answer and the X-ray background had just been discovered. The discovery of the Cosmic Microwave Background Radiation in 1965 changed all that and led to a remarkable series of space observatories in which studies of the origin and properties of the background radiation have been primary objectives.

In the late 1960s, Rashid Sunyaev and I wrote a survey of the background radiation at all wavelengths and the summary spectrum which we produced at that time is still one of my favorite diagrams - I apologise for showing it again (Figure 1). It brings back happy memories of a wonderful year spent working in Moscow. At that time, there were secure determinations of the background 


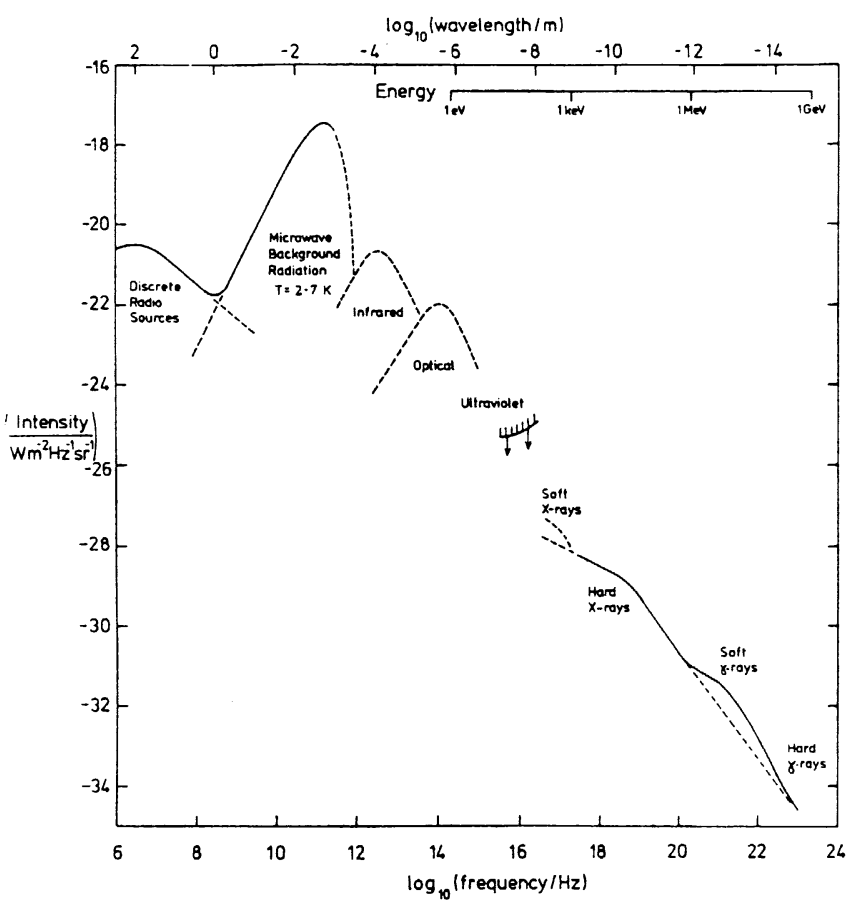

Figure 1. The spectrum of the isotropic extragalactic background radiation as it was known in 1969 (Longair and Sunyaev 1971). Dashed lines indicate regions in which the background was poorly constrained, those in the optical and infrared regions of the spectrum being no more than educated guesses.

radiation at long radio wavelengths, reasonably convincing evidence for the thermal nature of the microwave background radiation, good determinations of the shape of the X-ray background spectrum, including the bend at about $40 \mathrm{keV}$, and a background of hard $\gamma$-rays. Between about $1 \mathrm{~mm}$ and $0.1 \mathrm{keV}$, there were only observational upper limits. The situation was particularly bad in the wavebands from about 1 to $100 \mu \mathrm{m}$ where very little was known about either the background or the sources which might contribute to it. This did not stop us guessing what the background might be on the basis of the very fragmentary information available at that time. The dashed line in Figure 1 labelled 'Infrared' shows our guess - it is actually not so different from the present best estimates which we have just heard about at this meeting. This means we were either very clever, or more likely just very lucky! I prefer the latter interpretation.

By the time of the last IAU Symposium on the Background Radiation, the Heidelberg meeting of 1989 , there were new limits to the extragalactic background flux from IRAS observations in the wavelength range 12 to $100 \mu \mathrm{m}$, as well as limits in the optical waveband by Toller and new limits in the ultraviolet 
waveband from Bowyer and his colleagues (see Bowyer and Leinert 1990). These were summarised in my concluding remarks at that meeting (Longair 1990).

Before bringing that story up-to-date in the light of what we have learned at this meeting, it is worthwhile recalling where most of the background radiation originates. Suppose we have a distribution of sources, all with the same intrinsic luminosity $L\left(\nu_{0}\right)$ and with number density at the present epoch $N_{0}$. For simplicity, we assume that the sources have power-law spectra $L(\nu) \propto \nu^{-\alpha}$. It is then a straightforward calculation to show that the background intensity at frequency $\nu_{0}$ from redshift 0 to $z$ is

$$
I\left(\nu_{0}\right)=\frac{c}{H_{0}} \frac{L\left(\nu_{0}\right) N_{0}}{4 \pi} \int_{0}^{z} \frac{\mathrm{d} z}{\left(\Omega_{0} z+1\right)^{1 / 2}(1+z)^{2+\alpha}}
$$

For illustrative purposes, let us consider the simplest cases $\Omega_{0}=1$ and 0 with $\alpha=1$. It comes as a surprise to many astronomers to realise that half of the background radiation originates from remarkably small redshifts. For $\Omega_{0}=1$, half the background originates at redshifts less than $z=0.31$ and, for $\Omega_{0}=0$, from redshifts less than $z=0.42$. In other words, most of the background does not originate from inaccessibly large redshifts, but from redshifts which are well within the grasp of modern optical-infrared telescopes.

The evolution of the source population has to be very strong indeed to make a substantial difference to the background. I have given an example of this in Section 17.6 of my book Galaxy Formation (Longair 1998). The evolution of the quasar population can be represented roughly by a model in which the luminosity of each quasar increases as $(1+z)^{3}$ over the redshift interval $0<z<2$ and then remains constant at the value $27 L_{0}$ at all greater redshifts. Then, the background is only a factor of 5.4 greater than that expected if the sources did not change their luminosities with cosmic epoch.

\section{Selected Observational Highlights}

Considering first observations of the background itself, the highlights of the meeting for me were the following:

- The final products from the COBE mission: The final results of the COBE all-sky surveys in the millimetre, submillimetre and infrared wavebands were described by Hauser and are of outstanding importance for astrophysics and cosmology. I will say little about the observations of the Cosmic Microwave Background Radiation since that is a major industry in its own right and has been considered in detail at IAU Symposium No. 201 (Lasenby and Wilkinson 2001). Suffice to say that the determination of the spectrum of the radiation and its distribution over the sky have placed the conventional Big Bang picture of the early universe on a very secure observational footing. From the perspective of this meeting, the background measurements at shorter wavelengths also provide key data for astrophysical cosmology. Both Hauser and Puget emphasised the central importance of the extraction of the submillimetre background radiation due to dusty galaxies for the origin and evolution of galaxies. 
- The near infrared background: Direct observation of the background radiation at shorter wavelengths, say between 1 and $100 \mu \mathrm{m}$, is greatly hampered by the emission of zodiacal dust. Hauser reported upper limits to the background on the basis of the COBE observations. In the nearinfrared waveband from 1 to $4 \mu \mathrm{m}$, Matsumoto reported measurements of the background from the Japanese Near Infrared Spectrometer (NIRS) experiment. In fact, these observations are more or less coincident with the upper limits to the background reported by Hauser. It is of great importance to establish whether or not the measurements are in fact upper limits or a true measurement of the cosmic background in these wavebands.

- The Mid-infrared Waveband: In the waveband, $10 \leq \lambda \leq 100 \mu \mathrm{m}$, although the zodiacal background overwhelms the cosmological background, important upper limits, or even measurements, of the background are found from the spectrum of ultra-high energy $\gamma$-ray sources. Konopenko and Stecker reported remarkable observations of the $\mathrm{TeV}$ spectrum of the active galactic nucleus Markarian 501, which shows an exponential decrease in intensity at $\gamma$-ray energies greater than about $5 \mathrm{TeV}$. The cut-off is attribute to $\gamma-\gamma$ interactions between the high energy $\gamma$-rays and photons of the mid-infrared background radiation. Interpreted as upper limits to the background intensity in these wavebands, the background flux must be less than $\nu I(\nu)=10 \mathrm{nW} \mathrm{m}^{-2} \mathrm{sr}^{-1}$, a figure not very different from the upper limits observed in the optical waveband and similar to that inferred to be present in the 100 to $1000 \mu \mathrm{m}$ waveband from the COBE data. It is intriguing that this estimate of the mid-infrared background is about a factor of 50 lower than the direct upper limit derived from the COBE observations. The way forward is to repeat this type of observation for many more distant $\mathrm{TeV} \gamma$-ray sources. A straightforward test of this explanation for the high energy cut-off is that it should move to lower energies, the greater the path-length from the source to the observer.

- There was a ghost at the meeting, namely the observations of the optical extragalactic light attributed to Bernstein et al. (2000), who claim to have detected a cosmological background signal. So far as I can tell from what was reported, the measured intensity is similar to that reported by Toller (1990). These backgrounds are about twice the background found by summing the light from the counts of galaxies. Indeed, this factor of two problem would be present in the near infrared waveband as well if Matsumoto's observations represent a real measurement of the intensity of the background. Factors of two in this business are difficult to deal with, particularly when the extraction of the background from the observations is so difficult. If the factor of two discrepancy is real, it must be associated with faint diffuse structures which are not counted in the analyses of the Hubble Deep Field.

The data are summarised in Figure 2, which is a simplified and labelled version of the plot shown in Hauser's splendid review. It can be seen that the background spectrum in this most difficult wavelength region is gradually succumbing through these new observational approaches. 


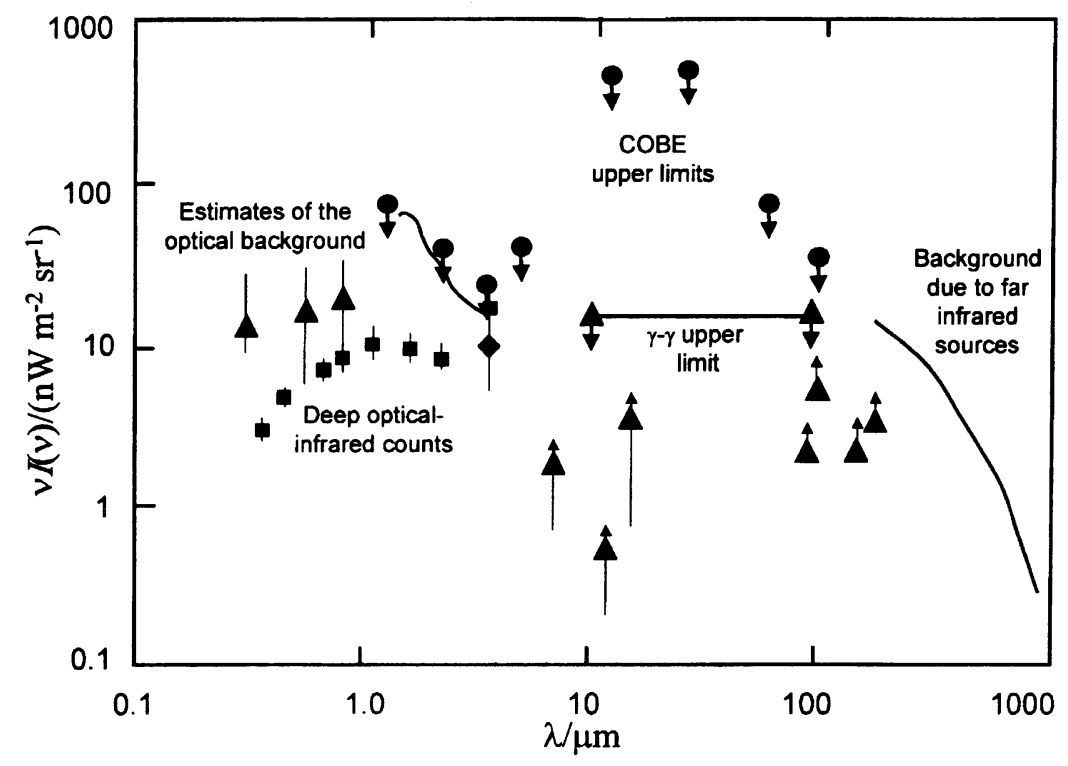

Figure 2. A schematic summary of the extragalactic background radiation from the optical to submillimetre waveband (after Hauser, this volume).

Where are the loopholes in these arguments and how can we do better? Ozernoy presented a remarkable review of his endeavours to model the zodiacal light emission and the complexities involved in constructing successful models. To the non-expert, the interesting point is the fact that the problem is very strongly time-dependent. It will be fascinating to see how well this type of modelling can help disentangle the cosmic signal from the local zodiacal component. Another major component needed to understand the total sky brightness is the modelling of the distribution of stars in the Galaxy. Cohen's comprehensive discussion of the completeness and accuracy of the modelling procedures was very impressive and seemed to leave few loopholes for changing significantly the contribution of stars to the foregrounds which have to be subtracted.

The zodiacal component is clearly the killer in this discussion and the obvious solution to the observational problem is to make observations of the background at such large distances from the Sun, say greater than $3 \mathrm{AU}$, that it does not make a significant contribution to the background intensity. Such a mission is on the menu of potential NASA missions, but I understand that it is not considered to be a high priority programme at the present time. Nobody at this Symposium would doubt the importance of nailing down once and for all the background in the near and mid-infrared wavelengths, free from the zodiacal emission of our Solar System. If we really believe this is important, we must press the inclusion of such a mission in the NASA programme. 


\section{Number Counts}

The astrophysical goal of studies of the background radiation is to understand the nature of the sources which make it up and so the next obvious step is to compare the observed backgrounds with the predicted value found by integrating the number counts of objects in each waveband. Excellent reviews were presented of the current state of counts of extragalactic sources from the optical to the submillimetre regions of the spectrum.

- Pozzetti presented a splendid update of the present status of the number counts of galaxies in the optical and near-infrared region of the spectrum. As she pointed out, most of the background is not contributed by the very faintest galaxies, but rather by those with magnitudes about 22 to 24 , for which it is feasible to obtain spectra with 8-metre class telescopes. Furthermore, the number counts at the faintest magnitudes are strongly converging and so the total background due to known types of galaxy can be determined with some confidence. This is the origin of the 'factor of two problem' alluded to in the last section. It seems reasonable that most of the luminosity of the visible parts of galaxies in the optical and near infrared wavebands is already accounted for.

- Among the most beautiful new observations were the very deep number counts at $2.2 \mu \mathrm{m}$ obtained by the Japanese 8-m Subaru telescope which were presented by Totani. These are the deepest counts obtained to date and they demonstrate vividly the great power of the Subaru telescope for these types of study. They confirmed very convincingly the convergence of the $2.2 \mu \mathrm{m}$ counts at the faintest apparent magnitudes.

- Equally impressive were the counts of sources at $15 \mu \mathrm{m}$ obtained from the ISO deep surveys at $15 \mu \mathrm{m}$ which were discussed by Cesarsky and Puget. This represents a triumph of image processing by the ISO science team. Equally impressive was the extension of the $15 \mu \mathrm{m}$ number counts to even fainter magnitudes through the use of gravitational lensing by rich clusters of galaxies by Metcalfe and his colleagues. The net result is that the number counts at $15 \mu \mathrm{m}$ are well defined at this wavelength and they show an excess of faint objects, as well as a decline at the very faintest flux densities. This is a familiar pattern, but it is intriguing to find it occurring also at mid-infrared wavelengths. This is just the beginning of the story since the next essential step is to understand the nature of the objects responsible for the $15 \mu \mathrm{m}$ emission.

- ISO also carried out observations of cosmological importance at wavelengths of about $100 \mu \mathrm{m}$ and these were described by Lemke. These observations are of special importance in conjunction with the counts of sources at submillimetre wavelengths since they are likely to be part of the same source population .

- Finally, the counts of sources at submillimetre wavelengths were described by Rowan-Robinson. This is one of the most gratifying stories for me personally, since Andrew Blain and I had expected that, because of the 
large negative K-corrections, there would excellent chances of detecting a large population of distant star-forming galaxies in the submillimetre waveband. Further, once a class of galaxy had been detected at redshifts $z \sim 1$, they would be readily observable out to redshifts $z \sim 10$. When the first deep submillimetre surveys were carried out by SCUBA three years ago, the population turned up in even greater numbers than our most optimistic predictions suggested. Michael Rowan-Robinson played a leading role in our consortium's observation of the Hubble Deep Field at $850 \mu \mathrm{m}$ with SCUBA on the JCMT and it is one of my favorite astronomical pictures. In this case, as described by Hauser and Puget, the total background intensity already contributed by discrete sources amounts to about half the background and so there cannot be much more to be observed at this wavelength. There are, however, problems in identifying precisely what the sources are and their redshifts. Because of the low angular resolution of the SCUBA observations, these need to be supplemented by radio and optical observations and it ends up being a very time-consuming job to make a reliable identification and secure a redshift. The sense of many of the successful identifications is that these objects are indeed galaxies at large redshift and as such are of prime importance in understanding galactic evolution and the star formation history of galaxies.

These results represent outstanding progress in opening up the mid- and far-infrared wavebands for cosmology, but it must be emphasised that these studies are only in their infancy. There is an urgent need to understand the astrophysical nature of the populations of objects being studied in the different wavebands. How uniform are the populations in space and with cosmic epoch? To determine these key features, it is essential to measure accurate positions for the sources, identify them securely and then find means of determining their redshifts. To achieve this, the full battery of ground-based observing facilities in the optical and radio wavebands, as well as the new space missions for the near to far-infrared wavebands are essential. Another key facility will be the Atacama Large Millimetre Array, ALMA, which will do for submillimetre astronomy what the HST has done for optical astronomy. Its high angular resolution and great sensitivity will enable accurate positions, and possible redshifts, to be determined for the faintest submillimetre sources.

\section{The Source Populations and the Modelling of their Evolution with Cosmic Epoch}

One of the key aspects of interpreting the counts and the background radiation is the creation of robust template spectra for the sources. Examples of the level of complexity likely to be necessary to explain the observations were presented by Helou, Franceschini and Rowan-Robinson. This is a far from trivial activity because as yet the samples of sources available for study throughout the entire near to far infrared waveband are limited and generally only broadband spectra are available. The obvious concern is the extent to which these templates are universal. Examples of the types of observation which makes life more difficult are: 
- Whereas many of the luminous far-infrared galaxies exhibit PAH features between 7 and $15 \mu \mathrm{m}$, the most luminous sources, the ULIRGs, do not seem to show PAH features. What exactly is going on?

- How many of the luminous far-infrared galaxies are powered by active galactic nuclei and how many are simply hyperactive star-forming galaxies? Both Sturm and Mushotzky suggested that about 10-20\% of the ULIRGs are powered by Active Galactic Nuclei. We need to remember, however, that Veilleux and his colleagues (1997) have shown that the fraction of luminous infrared galaxies powered by such nuclei is quite a strong function of far-infrared luminosity. These complications need to be part of the modelling process.

- It is really important to carry out detailed studies of a wide range of the nearest examples of active galactic nuclei to find out directly for these galaxies what the origin of the far-infrared luminosity is likely to be. My suspicion is that this story is more complex than we would expect and it provides further complications for the modellers.

- Finally, I infer from the wealth of data presented at this meeting that the physics of the objects which are important at different infrared wavelengths can vary quite widely. There is no reason why the evolution functions which describe these different physical processes should be the same - I believe the only safe thing to do is to treat each half-decade of waveband as separate source populations.

The importance of the various endeavours to model the infrared source populations is that they are indicative of the types of observation which are likely to be most fruitful in future large survey campaigns and for the scientific opportunities provided by the next generation of infrared observatories. It is interesting to compare the situation with that in radio astronomy long ago. The overall modelling of the source populations is still a useful exercise, but all the real science is now coming out of surveys for which the redshift data are complete. This has to be the major objective of the surveys which are currently underway. Unfortunately, it is not an easy business. The current angular resolution of mid and far infrared telescopes is too low to make identifications directly with very distant, and consequently very faint, optical objects. To make matters worse, if the objects are really dusty, they may be all the more difficult to detect even with telescopes as powerful as the VLT. I am not, however, pessimistic. Once the astrophysics of the more nearby objects are better understood, I am sure they will provide the clues as to how to proceed with future surveys. I find it reassuring that the observed number counts of faint submillimetre sources exceeded the most optimistic predictions which Andrew Blain and I made in 1993 - sometimes nature is on our side.

\section{Metals, Star Formation and the Extended Harwit Diagram}

The physics of the counts of sources and the background radiation in the infrared wavebands is intimately tied up with issues concerning the rates of star and metal 
formation. These issues were described from somewhat different perspectives by Savaglio and Cowie. The key issues in these areas relate to the rate at which heavy elements are built up as a function of cosmic epoch, for example, the splendid example of the build up of zinc, which is assumed to be a good tracer of the overall build up of elements such as iron and which is not strongly affected by absorption onto dust grains. Likewise, how reliable are the estimates of the changes in the cosmic abundance of diffuse atomic hydrogen with cosmic epoch? At first sight, it is very encouraging that, as the primordial hydrogen is condensed into stars and a certain fraction converted into heavy elements, the cosmic abundance of diffuse hydrogen seems to decrease roughly as expected. These observations, however, disguise a number of crucial questions about how the heavy elements are dispersed from the sites where they were created to the diffuse intergalactic gas. As Cowie pointed out, it is remarkable that even in neutral hydrogen absorbers, which are less dense than the mean intergalactic gas density, the enrichment of the heavy elements seems to have been effective.

Lilly and Madau tackled directly the problem of the global history of star-formation, one of the more exciting developments of the last few years. It is generally agreed that, at large redshifts, the global star formation rate derived from optical observations is significantly incomplete and it is encouraging that, once corrections for this incompleteness are made, the ultraviolet and submillimetre variations of the star-formation rate with cosmic epoch seem to be converging. However, it is still unclear precisely how the star-formation rate has changed with cosmic epoch at large redshifts. This is a key problem since the early formation of the heavy elements is likely to be important in seeding subsequent generations of star formation.

These considerations lead naturally to the pleasant diagram which Harwit showed in his introductory survey. There is a complex network of interactions between the birth, life and death of stars and these can be addressed uniquely by observations in the infrared waveband. In Figure 3, I have created what might be termed an extended version of the Harwit diagram to show how these topics are related to the processes of galaxy formation, as well as their evolution - indeed, the distinction between these terms is probably only a matter of taste.

Considering first the original Harwit diagram, many of the essential tools needed to treat different parts of the diagram were clearly described by Truran and Dwek. The essential processes needed to create the heavy elements were beautifully summarised by Truran and this provides the palette of physical processes needed by the astrophysical cosmologist to construct realistic models of the history of star and element formation. This seemed easy compared with the potential complexities of understanding the formation and destruction of dust grains described by Dwek. The key point is that dust is omnipresent in the universe of galaxies and there is every reason to believe that the properties of the dust may vary from one location to another. There is no question in my mind about the importance of what Dwek was telling us - we need a much more profound understanding of the nature of interstellar dust grains, if we are to use them as other than thermodynamic transformers.

This suggests that we are far from a detailed implementation of many ingredients of the Harwit diagram and that, in the immediate future, the best we can probably hope for are global approximations to what may be going on. 


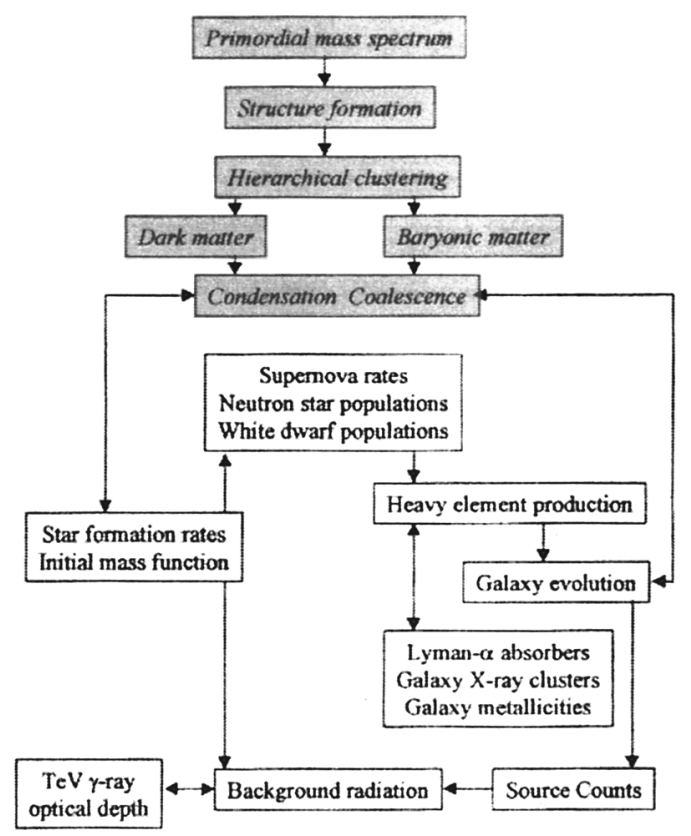

Figure 3. The extended Harwit diagram. The extended components of the diagram are indicated in italic type on a hatched background.

The approach taken by Silk in attempting to synthesise star and element formation issues with theories of the origin of galaxies and the large-scale structure of the Universe analytically seems to me to be very fruitful. I have indicated on the extended parts of the Harwit diagram how the issues of galaxy and structure formation can be incorporated into an even grander picture. What I find compelling is the diagram first published by Efstathiou and Rees (1988), and revised by Efstathiou (1995), which shows how the mass spectrum of galaxies and larger systems evolves with redshift according to the simplest version of the Press-Schechter formalism for hierarchical structure formation, which turns out to be a remarkably successful description of the results of supercomputer simulations, as well as giving a valuable intuitive picture for the preferred picture of structure formation (Figure 4). The diagram shows how the mass spectrum of systems of different mass evolves over the redshift range 0 to 10 , which is well within the range of observational capabilities in the optical and submillimetre waveband. There are very significant changes in the number densities of massive systems over this redshift range, which is the same as that over which most of the star-formation activity in galaxies must have taken place. Let me give some impression of how these ideas might be synthesised into the picture for star and element formation. 


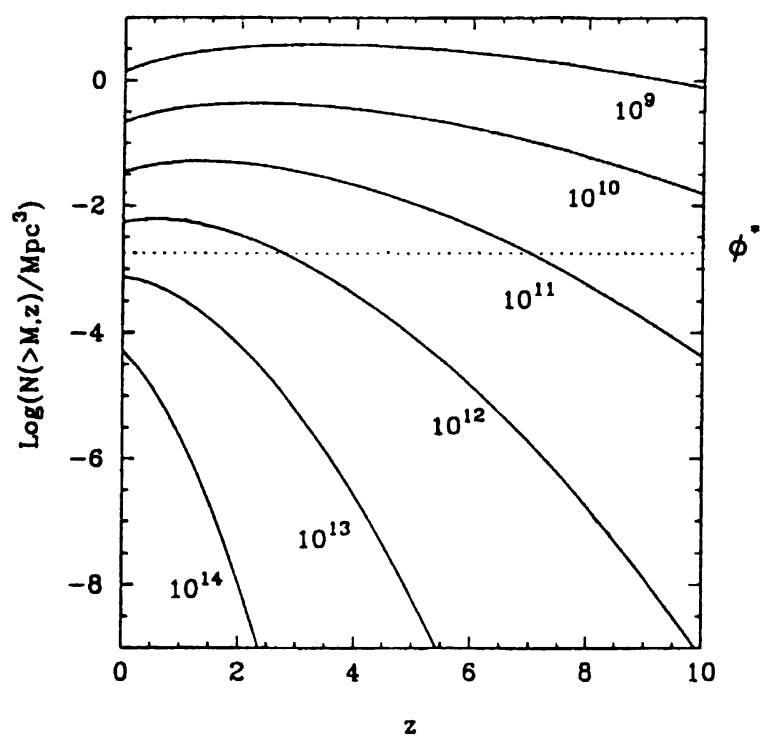

Figure 4. The evolution of the mass spectrum of bound objects with redshift found from the Press-Schechter function (Rees and Efstathiou 1988, Efstathiou 1995).

\section{Star Formation and Galaxy Formation}

Despite the well-rehearsed problems of interpreting observations of the evolution of the abundance of the elements with cosmic epoch, let me proclaim my allegiance to the approach taken by Pei and Fall (1995), described by Fall at this meeting (see also Pei et al. 1999). As he has emphasised, provided averages are taken over a large enough sample of damped Lyman- $\alpha$ absorption systems, all projection effects and problems of different lines of sight through different regions of the absorbing clouds average out and produce a meaningful average abundance. In their important paper of 1995, Pei \& Fall showed how dust plays a dominant role in the interpretation of data on the cosmic abundances of the elements. It affects almost every stage of the analysis from the completeness of the quasar samples to begin with, to the effects of extinction upon the abundances themselves. This is one of the reasons why submillimetre observations of the global star-formation rate are so important - extinction is unimportant in the selection of the galaxies and the estimates of the star formation rates.

Allon Jameson, Andrew Blain and I have shown recently how the equations of cosmic chemical evolution can be combined with the Press-Schechter formalism to produce a rather nice result which indicates clearly where the key physics lies in understanding the cosmic evolution of the chemical elements and the cosmic star formation rate. I will summarise our argument here briefly (more details are given in Longair (2000)). 
The evolution of the Press-Schechter mass function,

$$
N(M)=\frac{\bar{\varrho}}{\sqrt{\pi}} \frac{\gamma}{M^{2}}\left(\frac{M}{M^{*}}\right)^{\gamma / 2} \exp \left[-\left(\frac{M}{M^{*}}\right)^{\gamma}\right]
$$

with cosmic epoch is controlled by the value of the reference mass $M^{*}(z)=$ $M^{*}(0)[\delta(z) / \delta(0)]^{2 / \gamma}$, above which the mass spectrum cuts off exponentially. $\delta(z)$ is the function which describes the growth of linear perturbations in an expanding Universe and $\gamma=1+(n / 3)$, where $n$ is the spectral index of the power spectrum of initial density perturbations, $P(k)=k^{n}$ (for a simple derivation of these results, see Longair (1998)). We had previously derived a simple analytic expression for the merger rate of galaxies from the Press-Schechter mass function (Blain \& Longair 1993a,b; Blain et al. 1999).

In order to derive an expression for the luminosity density per unit comoving volume due to star formation during mergers, assumptions need to be made about the amount of energy liberated by star formation activity. In our 1993 approach, it was assumed that a fixed fraction $x$ of the total masses of the merging galaxies $M$ was converted into stars, liberating $0.007 x M c^{2}$ of energy, and resulting in the formation of a mass $x M$ of heavy elements. It is more realistic to allow $x$ to vary as a function of redshift, $x(z)$ and so we write $x(z)=$ $x_{0} \varepsilon_{*}(z)$, introducing a star formation efficiency $\varepsilon_{*}(z)$, normalized to unity at $z=0$. The luminosity density at redshift $z$ is then,

$$
\rho_{\mathrm{L}}(z)=0.007 x_{0} \varepsilon_{*}(z) c^{2} \int_{0}^{\infty} M \dot{N}_{\mathrm{form}} \mathrm{d} M .=0.007 \bar{\rho} \varepsilon_{*}(z) c^{2} \frac{2}{\gamma} \beta \frac{\dot{\delta}(z)}{\delta(z)}
$$

where $\beta=\phi x_{0} / \sqrt{\alpha} ; \phi$ and $\alpha$ are constants with values close to unity. In an Einstein-de Sitter model $\dot{\delta}(z) / \delta(z)=H_{0}(1+z)^{3 / 2}$.

We adopt the simplest form of the equations of cosmic chemical evolution given by Pei \& Fall (1995) for the closed box model, which can be expressed as $\dot{\Omega}_{*}+\dot{\Omega}_{\mathrm{g}}=0$, in which the density parameters $\Omega_{*}$ and $\Omega_{\mathrm{g}}$ refer to the stellar and gaseous components of the galaxies respectively. For the closed box model, $\Omega_{*}+\Omega_{\mathrm{g}}=\Omega_{\mathrm{g} \infty} . \Omega_{\mathrm{g} \infty}$ is the initial density parameter of primordial gas - at large redshifts, all the baryons were in the form of gas and there were no stars.

Some assumption about the dependence of the star formation efficiency $\varepsilon_{*}$ upon the density of gas and metals must be adopted. It is reasonable to assume that it is proportional to the product of the average density of gas $\Omega_{\mathrm{g}}$, representing the availability of fuel for star formation, and the average density of heavy elements synthesized by a given epoch $\Omega_{\mathrm{m}}$ - the latter represents the efficiency of cooling, mediated by the optically thin emission from dust and atomic fine-structure transitions of heavy elements $(\mathrm{Si}, \mathrm{O}, \mathrm{N}$ and $\mathrm{C})$ in dense molecular clouds. Under these assumptions, we can write $\varepsilon_{*}(z)=k \Omega_{\mathrm{g}} \Omega_{\mathrm{m}}$, where $k$ is a constant. This is a Schmidt star formation law (Kennicutt 1998).

More details of these calculations are given in Longair (2000) - the result is an analytic expression for the star formation efficiency

$$
\varepsilon_{*}=a \operatorname{sech}^{2}\left[b J(z)-\cosh ^{-1} \sqrt{a}\right] .
$$


where the constants $a$ and $b$ have the values, $a=\Omega_{\mathrm{g} \infty}^{2} k Q / 4$ and $b=\beta k \Omega_{\mathrm{g} \infty} / \gamma$, while the function $J(z)$ is

$$
J(z)=\int_{0}^{z} \frac{\dot{\delta}}{\delta} \frac{\mathrm{d} r}{\mathrm{~d} z^{\prime}} \frac{1}{c\left(1+z^{\prime}\right)} \mathrm{d} z^{\prime} .
$$

In an Einstein-de Sitter world model $\mathrm{d} r / \mathrm{d} z=\left(c / H_{0}\right)(1+z)^{-3 / 2}$, and so $J=$ $\ln (1+z)$ and the solution is analytic,

$$
\varepsilon_{*}=a \operatorname{sech}^{2}\left[b \ln (1+z)-\cosh ^{-1} \sqrt{a}\right] .
$$

These simple formulae completely define the form of the star formation rate as a function of epoch. The magnitude of $a$ determines the height of the peak of the star formation efficiency as compared with its value at $z=0$, while the ratio of $a$ and $b$ determines the redshift of the peak. The maximum occurs when the argument of the $\operatorname{sech}^{2}$ function is zero, that is at,

$$
z=\exp \left(\frac{\cosh ^{-1} \sqrt{a}}{b}\right)-1,
$$

for an Einstein-de Sitter model. In this model $\varepsilon_{*} \propto(1+z)^{-2 b}$ at large redshifts, and, $\varepsilon_{*}=(1+z)^{2 b \sqrt{1-a^{-1}}}$, at small redshifts. The star formation rate for the Einstein-de Sitter model, normalised to its value at the present epoch, is therefore,

$$
\dot{\Omega}_{*}=(1+z)^{3 / 2} a \operatorname{sech}^{2}\left[b \ln (1+z)-\cosh ^{-1} \sqrt{a}\right] .
$$

In Figure 5, examples of the forms of the star formation rate with cosmic epoch have been derived by fitting the expression (4) to observations of the background spectrum derived by Fixsen et al. (1998) and the $60 \mu \mathrm{m}$ IRAS number counts (Bertin et al. 1997) for different assumed temperatures of the emitting dust grains. For comparison, various direct estimates of the star formation rate are shown. The derived curves provide a satisfactory envelope for the observations, recalling that the vertical normalisation is arbitrary.

Once the expression (3) for the global star formation rate has been established, it is straightforward to work out the variation of the density of metals, the gas density, the metallicity and the build up of the heavy elements in the intergalactic gas as functions of redshift (see Longair 2000). We have shown that the resulting expressions can provide a good account of the observed variations of these quantities with cosmic epoch.

How seriously should we take these models? The same type of formalism can be made to work for a wide variety of cosmological models. The key feature of the calculations is that the formation of stars takes place through the conversion of diffuse intergalactic gas into stars in galaxies. In the present calculations, one of the most important features concerns the assumptions made about the dependence of the efficiency of the star formation rate upon density and metallicity and that must be present in all similar approaches. Nonetheless, I would recommend the $\operatorname{sech}^{2}$ formula (3) for the efficiency of star formation to you. It has the pleasant feature of approximating to power-laws in $(1+z)$ on either side of the redshift at which it takes a maximum value. 


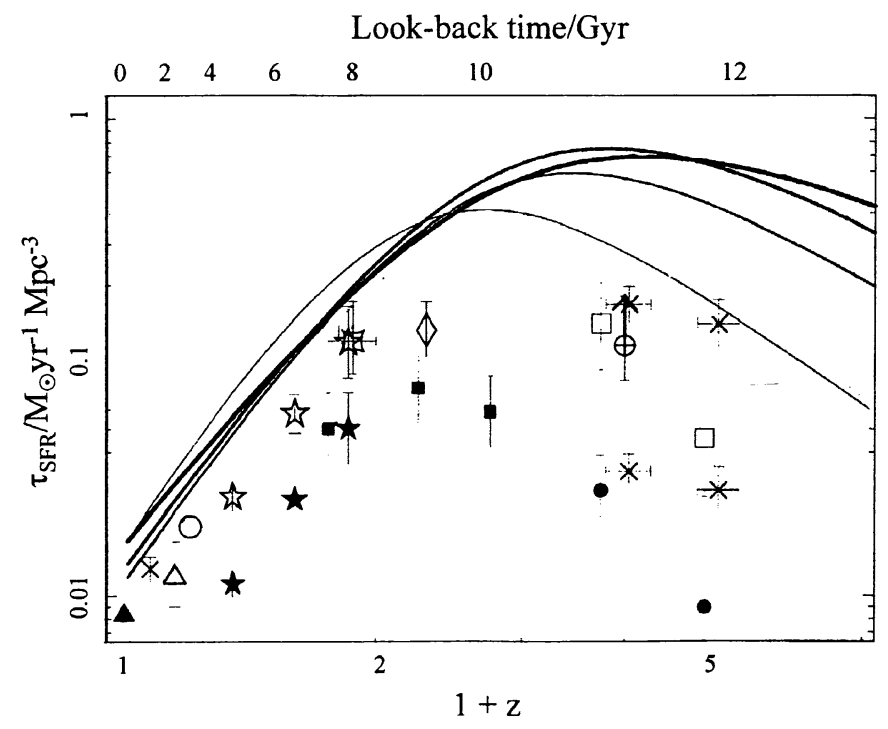

Figure 5. The star formation histories derived for the best-fitting models with dust temperatures $T_{\mathrm{d}}=35,40,45$ and $50 \mathrm{~K}$ (solid lines in order of increasing thickness). The vertical normalization of the curves is arbitrary.

\section{The Future}

As Zeldovich told us in 1973, 'A platitude must be stated with force and clarity.' My platitude is 'we live in exciting times'. There has been a revolution in astrophysical cosmology with the ability to make direct observations of the distant Universe throughout the 0.1 to $1000 \mu \mathrm{m}$ waveband. This must be the first conference at which such a statement could be made with a straight face. On the last day of the meeting, we heard of many future plans for powerful observing facilities which will undoubtedly answer many of the thorny problems discussed at this meeting, as well as raising a host of new ones.

The prospect is remarkable. In ground-based astronomy, the epoch of the 8-10 metre telescopes is upon us and there is the realistic possibility of building even larger optical-infrared telescopes. The next major breakthrough from the ground will undoubtedly take place with the construction of the Atacama Large Millimetre Array, ALMA, which I regard as the most exciting international development in astronomy. In space, there is the prospect of a remarkable series of observatories: MAP, ASTRO-F, SIRTF, FIRST, Planck, NGST, ..., each of them contributing quite distinct science to the goal of understanding the origins of our galaxies, complete with their stars and element abundances.

Finally, let me make a plea for a background mission to measure the optical/infrared background free from the effects of zodiacal dust. My sincere thanks to all the organisers, and particularly to Martin Harwit, for organising this splendid Symposium. 


\section{References}

Bernstein, et al. 2000, ApJ, (in press)

Bertin, E., Dennefeld, M., \& Moshir, M. 1997, A\&A, 323, 685

Blain, A. W., Jameson, A., Smail, I., Longair, M. S., Kneib, J.-P., \& Ivison, R. J. 1999, MNRAS, 309, 715

Blain, A. W. \& Longair, M. S. 1993a, MNRAS, 264, 509

Blain, A. W. \& Longair, M. S. 1993b, MNRAS, 265, L21

Blain, A. W. \& Longair, M.S. 1996, MNRAS, 279, 847

Bowyer, S. \& Leinert, C., eds. 1990, The Galactic and Extragalactic Background Radiation. (Dordrecht: Kluwer Academic Publishers)

Efstathiou, G. 1995, in Galaxies in the Young Universe, eds. H. Hippelein, K. Meisenheimer, \& H.-J. Röser (Berlin: Springer-Verlag), 299

Efstathiou, G. \& Rees, M. J. 1988, MNRAS, 230, 5

Fixsen, D. J., Dwek, E., Mather J. C., Bennett, C.L., \& Shafer, R. A. 1998, ApJ, 508,123

Kennicutt, R. C. 1998, ApJ, 498, 541

Lasenby, A. \& Wilkinson, A. (eds) 2000 New Cosmological Data and the Values of the Fundamental Parameters. IAU Symp. No. 201. (San Francisco: ASP)

Longair, M. S. 1990, in The Galactic and Extragalactic Background Radiation, eds. S. Bowyer \& C. Leinert (Dordrecht: Kluwer Academic Publishers), 469

Longair, M. S. 1998, Galaxy Formation, (Berlin: Springer-Verlag)

Longair, M. S. 2000, in AIP Conf. Proc., 516, 26th International Cosmic Ray Conference, Salt Lake City, eds. B. L. Dingus, D. B. Kieda \& M. H. Salamon (New York: AIP), 3

Longair, M. S. \& Sunyaev, R. A. 1971, Uspekhi Fiz. Nauk., 105, 41. [English translation: Soviet Physics Uspekhi, 14, 569]

Pei, Y. C. \& Fall, S. M. 1995, ApJ, 454, 6

Pei, Y.C., Fall S. M., \& Hauser M. G. 1999, ApJ, 522, 604

Press, W.H. \& Schechter, P. 1974, ApJ, 187, 425

Toller, G. 1990, in The Galactic and Extragalactic Background Radiation, eds.

S. Bowyer \& C. Leinert (Dordrecht: Kluwer Academic Publishers), 21

Veilleux, S., Sanders, D. B., \& Kim, D.-C. 1997, ApJ, 484, 92 